LA-UR- $04-2709$

Approved for public release; distribution is unlimited.

\section{Submitted to:}

Author(s):

J. M. Taccetti, S. H. Batha, J. R. Fincke, N. D. Delamater, N. E. Lanier, G. R. Magelssen, and R. M. Hueckstaedt - LANL

S. D. Rothman, C. J. Horsfield, and K. W. Parker AWE, plc

Astrophysics and Space Science (Kluwer Academic Publishers), for Proceedings of the 5th International Conference on High Energy Density Laboratory Astrophysics, March 10-13, Tucson, AZ 


\title{
RICHTMYER-MESHKOV INSTABILITY RESHOCK EXPERIMENTS USING LASER-DRIVEN DOUBLE-CYLINDER IMPLOSIONS
}

\author{
J. M. Taccetti, S. H. Batha, J. R. Fincke, N. D. Delamater, N. E. Lanier, G. R. \\ Magelssen, and R. M. Hueckstaedt \\ Los Alamos National Laboratory, Los Alamos, USA
}

S. D. Rothman, C. J. Horsfield, and K. W. Parker

Atomic Weapons Establishment plc, Aldermaston, UK

\begin{abstract}
As a shock travels through the interface between substances of different densities, existing perturbations can grow via the Richtmyer-Meshkov (RM) instability. The study of the RM instability in a convergent geometry leads to a better understanding of implosions applicable to inertial confinement fusion and various astrophysical events, such as core-collapse supernovae. We present results of laser-driven doublecylinder implosions performed at the Omega laser facility with an emphasis on sending a second shock through an already shocked RM unstable interface. The uniform reshock of a cylindrical interface is achieved by inserting a second cylinder inside the first that reflects the inwardly travelling shock and causes it to interact a second time with the unstable interface. We present an analysis of the instability growth as a function of shock strength and zero-order perturbation behavior during reshock.
\end{abstract}

Keywords: Richtmyer-Meshkov, Omega laser facility, cylindrical implosion, experiment, simulation

\section{Richtmyer-Meshkov Instability Reshock and Astrophysical Processes}

The Richtmyer-Meshkov instability (RMI) drives the growth of perturbations at the interface between substances of different densities when a shock travels through it, regardless of whether the shock is traveling from a heavier to a lighter medium or vice versa. It promotes turbulence and mixing of the materials at either side of this interface. The RMI has relevance for a broad range of topics, including many astrophysical processes, sudh as RMI-induced mixing during the explosion phase of core-cottape (Type II SNe, shock waves generated by SNe interacting with compressed ISM or denser motecular clouds, vortex formation in ISM (which may affect evolutiokscenarios of stars and structure formation in nebulae), morphology of SNe remnants (e.g. Cygnus Loop), and shock-clump interaction (Takabe, 2001). Any of these processes could experience reshock as well. Reshock occurs when a second shock travels through an already shocked interface.

We study RMI reshock in cylindrical geometry, which includes convergent effects such as those seen in many astrophysical systems, but is still 2D and therefore simpler to diagnose and interpret than fully $3 \mathrm{D}$ spherical geometry. We can also use simulation codes to model these problems and in that way attempt to validate the codes and learn more about our experimental results.

This work is a subset of a larger campaign to study mix in cylindrical geometry, which includes work with singly shocked surfaces, both smooth and perturbed, and both axial and azimuthal periodic perturbations (Barnes et al., 2002, Lanier et al., 2003, Balkey et al., Fincke et al.). The principal aim of this campaign is to measure the RMI growth and mix during shock and reshock in a convergent geometry, and to use this knowledge to validate existing simulation codes.

\section{Experimental Method}

Our basic experimental method consists of imploding cylinders composed of concentric shells of different materials (commonly referred to as 'targets'), using multiple laser beams to drive the implosion. The laser energy deposited on the outer surface of the target blows off material, causing the target to implode, and also drives a cylindrically symmetric shock through the shells embedded in the target. The experiments (or 'shots') 
are performed at the OMEGA laser facility at the Univ. of Rochester (Boehly et al., 1997).

An $\mathrm{Al}$ shell in the target is shocked twice: first by the inward-traveling shock and then again by an outward-traveling reflected one. Double-cylinder targets were developed to study mixing due to this reshock in a more controlled manner. Compared to traditional single-shell targets, an inner core was added to make the reflected shock more uniform than if reflected from the origin. The existence of this inner core also shortens the time between shock and reshock, allowing us to capture more of the dynamics on our short diagnostic time-window.

A cross-section of the target and a pre-shot side-on radiograph are shown on Fig. 1. The target is approximately $2.5 \mathrm{~mm}$ long and $1 \mathrm{~mm}$ in diameter. The outer Al shell, or 'marker layer', is $500 \mu \mathrm{m}$ long, centered on the target. The inner core is centered axially with respect to the marker layer but is longer, extending $100 \mu \mathrm{m}$ beyond either end. Table 1 shows the nominal thickness of each layer of material. All the results presented in this paper were obtained using targets such as the one described, and all were shot during the Sept. 2003 campaign.

The double-cylinder target design shown evolved from previous ones and resulted in a significant improvement in data. Changes to the design included: denser foam between the $\mathrm{Al}$ marker and inner core to decrease shock velocity, and increased inner core diameter to make up for longer shock travel time, maintaining enough distance between marker and core to improve visibility of the mix region.

All the targets presented in this paper were so-called 'smooth' marker layer targets. No perturbations were added to the surfaces, and a sample surface was metrologized to be smooth to within $100 \mathrm{~nm}$ RMS. Follow-up experiments will investigate growth of surfaces with prescribed perturbations.

\begin{tabular}{|l|l|r|}
\hline layer & material & thickness \\
\hline \hline ablator & epoxy & $55 \mu \mathrm{m}$ \\
\hline \hline marker & $\mathrm{Al}$ & $8 \mu \mathrm{m}$ \\
\hline & $\mathrm{CH} 90 \mathrm{mg} / \mathrm{cc}$ foam & $310 \mu \mathrm{m}$ \\
\hline core & $\mathrm{Cu}$ & $5 \mu \mathrm{m}$ \\
\hline & acrylic & $115 \mu \mathrm{m}$ \\
\hline
\end{tabular}

Table 1. Nominal cylindrical target layer thicknesses.
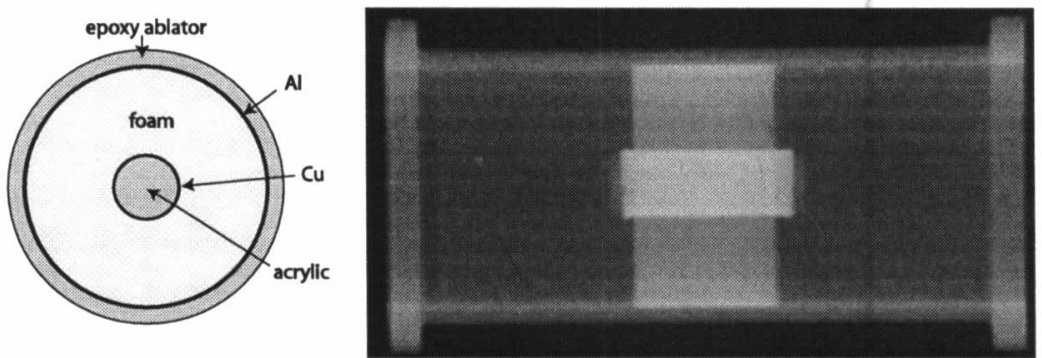

Figure 1. Cross-section of target and side-on radiograph.

It is instructive to follow the sequence of events that occur during a double-cylinder implosion. As explained in more detail by Graham et al. (Graham et al., 2000), a shock traveling from a lighter to a heavier fluid results in a transmitted shock (TS) and a reflected shock (RS), and a shock traveling from a heavier to a lighter fluid results in a TS and a reflected rarefaction wave. The laser beams drive a cylindrical shock inwards from the epoxy. This shock traverses the epoxy/Al (light to heavy) interface, resulting in a RS and a TS. The TS next traverses the $\mathrm{Al} /$ foam interface (heavy to light), resulting in a TS traveling inwards and a rarefaction wave traveling outwards. The TS travels through the foam until it crosses the foam/Cu interface (light to heavy), resulting in a RS and a TS. This reflected shock is the one that will reshock the marker layer. The TS entering the core rattles around resulting in multiple reflected and transmitted shocks. 


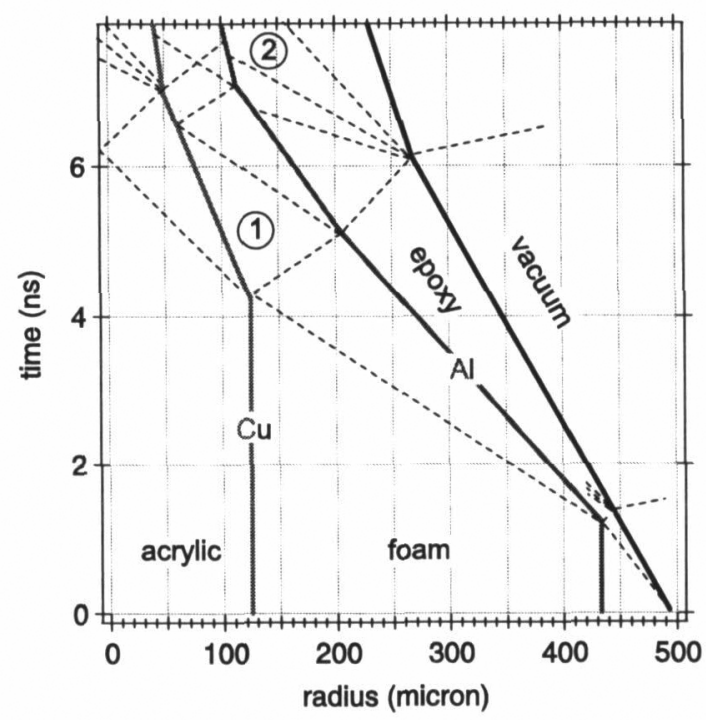

Fig. 2. Simplified $\mathrm{r}-\mathrm{t}$ diagram. Solid lines represent material interfaces ( $\mathrm{Al}$ and $\mathrm{Cu}$ layer thicknesses are ignored); dashed lines represent shocks. Effects at 1. and 2. are explained in the text.

A simplified r-t diagram can be used to explain two observed dynamic effects (see Fig. 2). The laser beams are on for $1 \mathrm{~ns}$, and $t=0$ is defined as the $2 \%$ rise point of the pulse. The $\mathrm{Al}$ and $\mathrm{Cu}$ layer thicknesses are ignored and shocks are followed. The shock velocities shown are estimated by using initial layer radii and approximate shockinterface interactions observed in our data, and by requiring that shocks travel faster in lighter media. The post-shock Al marker $v \sim 70 \mu \mathrm{m} / \mathrm{ns}$. The principal dynamic effects observed are: 1. Multiple shocks between the $\mathrm{Al}$ and $\mathrm{Cu}$ slow down, stop and reverse the implosion of the inner surface of the $\mathrm{Al}$ marker, and 2. A rarefaction wave propagating inward from the epoxy/vacuum interface causes the outer surface of the marker to expand.

\section{Data Analysis and Comparison with RAGE simulations}

Our principal diagnostic is an x-ray framing camera viewing the target axially. The camera uses a 4 -strip microchannel plate and a $4 \times 4$ pinhole array with $15 \mu \mathrm{m}$ diameter pinholes. The Fe backlighter, mounted on the opposite end of the target, is hit by 5 OMEGA laser beams ( $1 \mathrm{~ns}$ pulse time-delayed relative to the driving pulse) and produces $6.7 \mathrm{keV}$ x-rays. The strength of the signal recorded on film depends on the transmission through the target, which in turn depends on the materials in the target. The framing camera takes 16 frames, spanning a total of $1.2 \mathrm{~ns}$ ( 4 strips with 4 frames each, with 60 ps between frames within each strip, 300 ps between strips).

We measure the marker layer outer edge and inner edge, and the outer edge of copper coating on the acrylic core. A lineout of the angle-averaged transmission is obtained, and each edge is defined as the $50 \%$ intensity point between the local maximum and minimum intensity near that edge. Parallax errors (as the radiographs integrate through the entire target and the target is not perfectly aligned to all 16 camera pinholes) are taken into account and removed numerically (as in Lanier et al., 2003). The result of the analysis of four shots is shown in Fig. 3. Not every frame of each shot is analyzed some are dark due to the backlighter not being on and the fourth frame of every strip is dismissed due to camera operation which causes a longer than normal integration time for that frame.

Marker and core trajectories show implosion and re-expansion. The marker layer reaches a minimum width at $\sim 5.2 \mathrm{~ns}$, when the shock reflected from the core strikes the inner surface of the marker layer. The marker width then remains approximately constant for $\sim 1 \mathrm{~ns}$ during shock traversal. Maximum compression (minimum inner edge) of 
marker layer occurs at $\sim 6.8 \mathrm{~ns}$. At this point, the outer edge of the marker layer begins to move out. As stated earlier, we believe this may be the result of a rarefaction wave traveling inwards from the epoxy/vacuum interface.

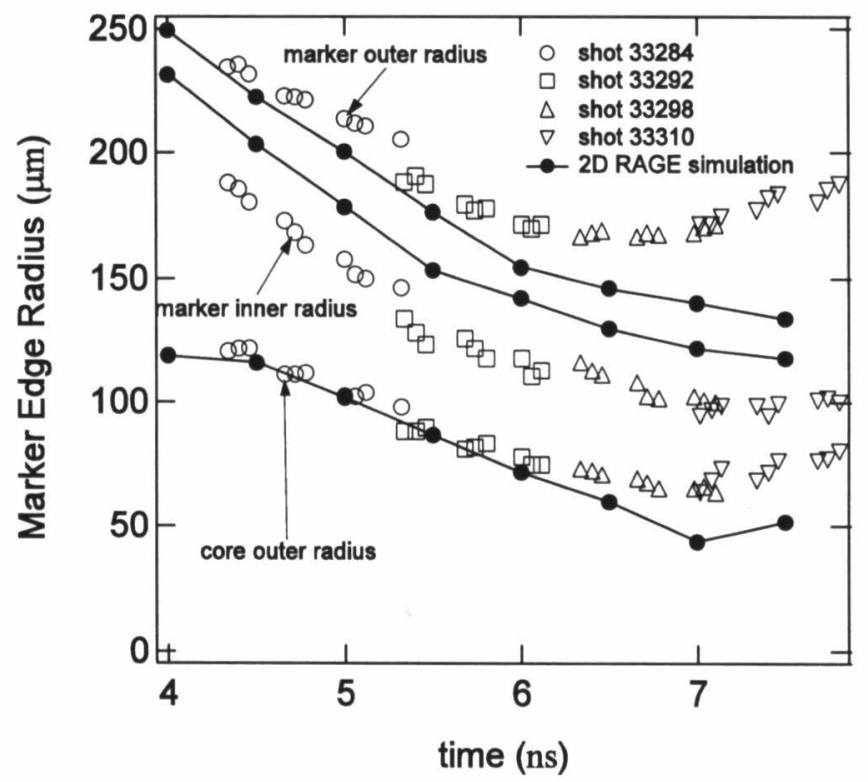

Figure 3. Comparison of experimental results and 2D RAGE simulation results.

The experiments were modeled with the RAGE code (Baltrusaitis et al. 1996). RAGE is a 3D multi-material Eulerian radiation-hydrodynamics code developed by LANL and SAIC. It features a continuous adaptive mesh refinement algorithm for following shocks and contact discontinuities with a very fine grid while using a coarse grid in smooth regions. It uses a second order Godunov type scheme. Laser deposition is not yet fully integrated into the code; it is calculated with LASNEX and implemented as an energy source in RAGE.

The calculations shown were performed in $2 \mathrm{D}$ and assuming a smooth marker layer. The calculated thickness of the marker layer does not agree with the data even if one were to add the instrumental blurring effects - which would add about $15 \mu \mathrm{m}$ to the marker width. Calculations also do not show the re-expansion of the outer surface of the marker layer. The reasons for this disagreement are still under investigation. On the other hand, RAGE gets the timing right: the shock strikes the core between 4 and $4.5 \mathrm{~ns}$, reshock of the marker layer occurs $\sim 5.5 \mathrm{~ns}$, and expansion of the core begins at $\sim 7 \mathrm{~ns}$.

\section{References}

Balkey, M.M., Batha, S.H., Lanier, N.E. et al.: in preparation.

Baltrusaitis, R.M., Gittings, M.L., Weaver, R.P., et al.: 1996, Simulation of shock-generated instabilities, Phys. Fluids 8, 2471-2483.

Barnes, C.W., Batha, S.H., Dunne, A.M. et al.: 2002, Observation of mix in a compressible plasma in a convergent cylindrical geometry, Phys. Plasmas 9, 4431-4434.

Boehly, T.R., Brown, D.L., Craxton, R.S. et al.: 1997, Initial performance of the OMEGA laser system, Opt. Commun. 133, 495-506.

Fincke, J.R., Lanier, N.E., Batha, S.H. et al.: 2004, Postponement of saturation of the Richtmyer-Meshkov instability in a convergent geometry, to be published.

Graham, M.J, and Zhang, Q.: 2000, Numerical simulations of deep nonlinear Richtmyer-Meshkov instability, Astrophys. J. Suppl. Ser. 127, 339-346.

Lanier, N.E., Barnes, C.W., Batha, S.H. et al:: 2003, Multimode seeded Richtmyer-Meshkov mixing in a convergent, compressible, miscible plasma system, Phys. Plasmas 10, 1816-1821.

Takabe, H.: 2001, Astrophysics with intense and ultra-intense lasers "laser astrophysics", Prog. Theor. Phys. Suppl. (Japan) 143, 202-265. 